\title{
Measurements in OLPC Mesh Networks
}

\author{
Vaibhav Rastogi, Vinay J. Ribeiro and Aditya D. Nayar \\ Computer Science and Engineering \\ Indian Institute of Technology Delhi \\ New Delhi, India 110016 \\ Email: \{vaibhavrastogi,vinay\}@cse.iitd.ernet.in, aditya.nayar@gmail.com
}

\begin{abstract}
The ease of deployment and the self configurable aspect of wireless mesh networks has generated lot of interest in them during the recent years. The One Laptop per Child (OLPC) project uses mesh networking, closely adhering to IEEE 802.11s WLAN mesh standard, to provide networking in a classroom environment. Our work measures and analyzes the performance of OLPC mesh networks. It includes several experiments concerning performance of UDP and TCP across a range of network topologies. Our experiments provide useful information about several aspects of these networks, including connectivity range, effect of RTS/CTS and Tx/Rx capabilities.
\end{abstract}

\section{INTRODUCTION}

A wireless mesh network (WMN) is a multihop ad hoc network where each node behaves as a router [1]. Such networks typically possess self organizing, self healing and self configuring capabilities. This provides robustness, reliability and easy of maintenance to wireless mesh networks. As a result WMNs are becoming popular for numerous applications.

In this paper we study the performance of the One Laptop Per Child (OLPC) networks. The OLPC [2] project uses IEEE 802.11s mesh networking to provide networking in a classroom environment. The project uses low-cost OLPC XO laptops provided to students and OLPC XS computers that act as school servers. The rugged, low-power XOs contain flash memory instead of a hard drive and use Linux as their operating system. Networking is provided by the Marvell 88w8388 wireless chipsets and 88w8015 RF transceivers. Libertas, the firmware for the wireless chipsets closely follows IEEE 802.11s draft standard.

IEEE 802.11s [3] is an amendment, currently in the draft stage, to the IEEE 802.11 standard, defining protocols for mesh networking. An 802.11s mesh networks has nodes called mesh points which can form mesh links with each other. Some nodes are designated as mesh portals. They act as gateways providing access to the outside world. Mesh routes are formed using a routing protocol called the Hybrid Wireless Mesh Protocol, or HWMP. HWMP is based on AODV [4] and tree-based routing. Vendors may include their own routing protocols apart from a mandatory implementation of HWMP. The underlying MAC and PHY mechanisms are that of $802.11 \mathrm{~g}$.

This paper covers work done in February-July, 2008. In all our experiments we employ the $\mathrm{B} 2$ test version of the $\mathrm{XO}$ hardware, version 703 of the OLPC build, version Q2D14 of OLPC firmware, and WLAN firmware version 5.110.22.p1. The WLAN firmware is proprietory and closed source. Note that the newer versions the above since developed may offer better capabilities than those we document here.

We measure throughput using Iperf, a bandwidth measurement tool [5]. Iperf provides for both UDP and TCP measurements allowing one to use different parameters, like the sending data rate, packet size, TCP window size, UDP buffer size among others. Most indoor throughput measurement experiments use a sniffer to sniff the channel for data frames in promiscuous mode. Unless explicitly mentioned, all results presented here employ plain CSMA, i.e., with the RTS/CTS mechanism disabled. At the start of the each experiment a free channel, as detected by a scan of channels from the wireless NICs themselves, in the $2.4 \mathrm{GHz}$ band is selected.

All multihop experiments use blinding tables. In such tables certain MAC addresses are blacklisted so that even if packets from these are received at the network interface they get discarded, effectively "blinding" the given XO to the blacklisted MAC addresses. By using blinding tables we ensure that certain links in the network are disabled, thus guaranteeing a particular desired routing topology.

The rest of the paper is organized as follows. In Section II we report results of outdoor experiments measuring how throughput varies with distance between OLPCs. We discuss results on multihop experiments in Section III. In Sections IV we study the impact of RTS/CTS on performance. Section V presents related work. Finally, we present our concluding remarks in Section VI.

\section{LINE OF SIGHT RANGE TESTS}

In this section we vary the distance between two OLPC nodes within line-of-sight of each other and measure throughput. These reachability experiments are useful in determining the performance of XOs in an out of class, for example, a playground, environment.

We used two OLPC XOs for range testing outdoors. The maximum range was checked using ping and we could get the laptops to communicate up to about 150 meters. We then tested throughput at a few discrete distances between zero and $150 \mathrm{~m}$.

Figure 1 is a Google Map of the location used for range testing experiments. The map represents a portion of the IIT Delhi campus. The black cross and circles represent the different approximate locations of the laptops during the tests. One laptop was kept fixed at position marked by cross, which 


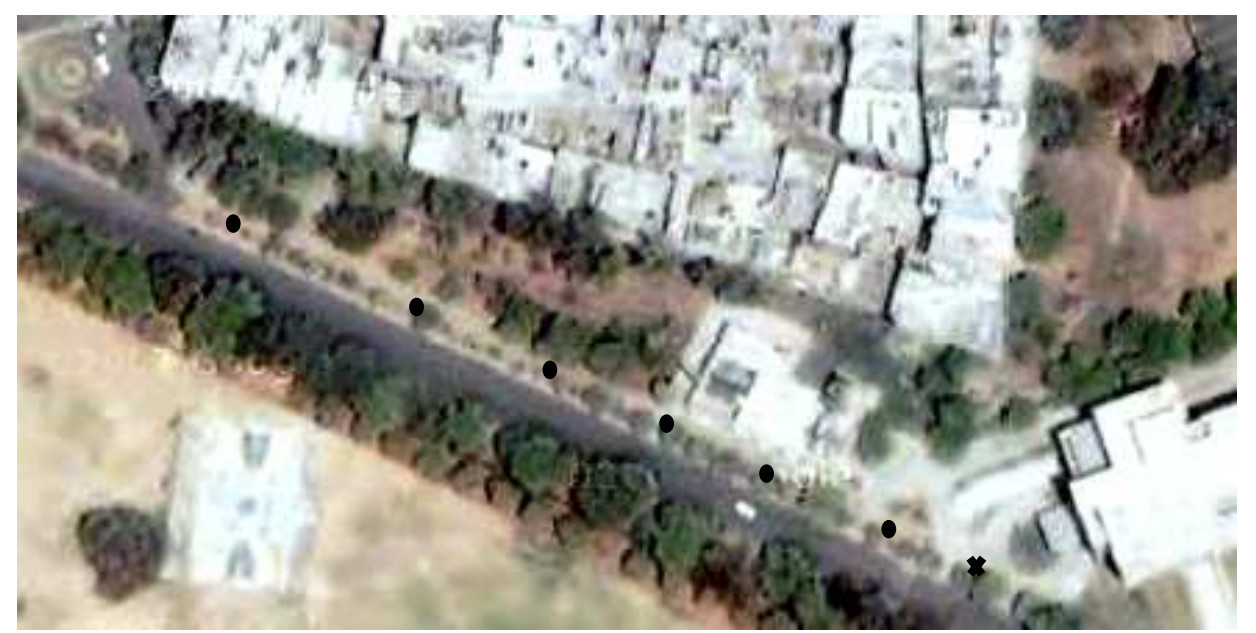

Fig. 1. The area for range testing experiments (map courtesy: Google Maps)

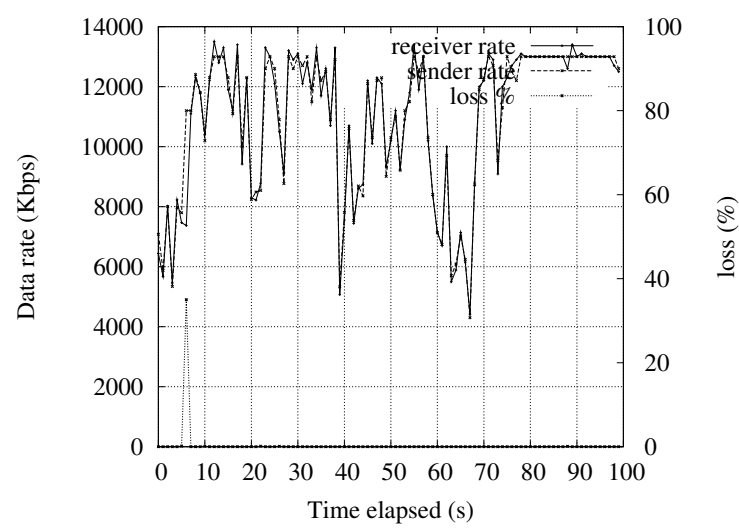

(a) UDP 13 Mbps data rate

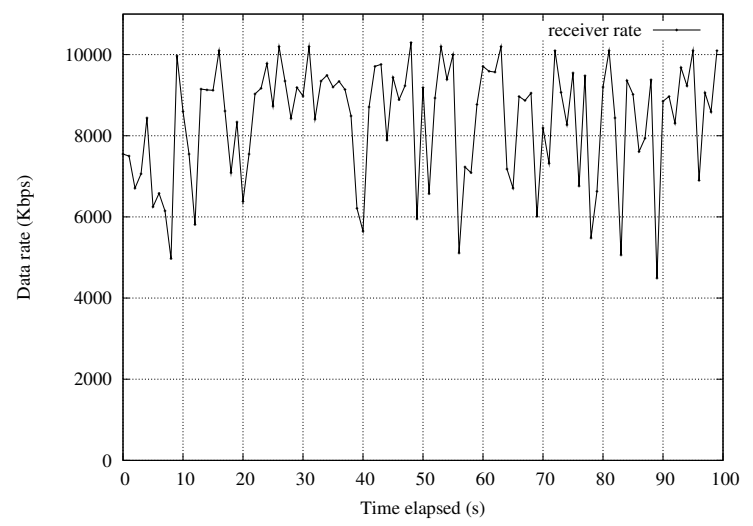

(b) TCP

Fig. 2. Plots for $16 \mathrm{~m}$ range

is in front of the Bharti Building where the Computer Science Department is located while the other laptop was kept on different green spots during different experiments. The farthest green spot is near the IIT hospital. During the experiments, the laptops were kept at approximately $1.2 \mathrm{~m}$ above ground so that line of sight is easy to maintain.

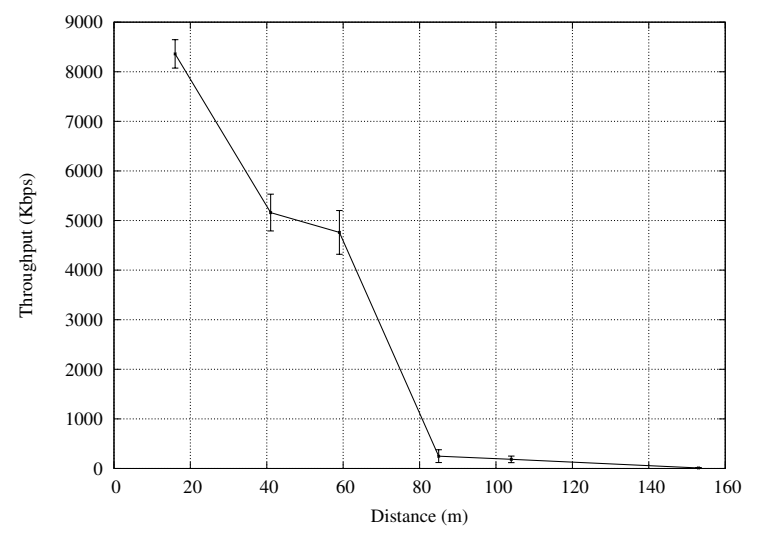

Fig. 3. TCP throughput variation with distance

Figure 2 depicts throughput of TCP and UDP at range of $16 \mathrm{~m}$. We observe that TCP throughput varies between 6-10Mbps over time. TCP's congestion control mechanism by design adapts its sending window to adapt to channel conditions. Hence such variation is to be expected.

We also observe that Iperf's UDP throughput varies between $7-13 \mathrm{Mbps}$. In this experiment, the UDP send-rate is set to $13 \mathrm{Mbps}$. However, Iperf checks the number of bytes actually written into the UDP socket after giving a socket write command in order to help regulate the sending rate at the application layer. As a result there is an implicit feedback mechanism due to which the effective UDP sending rate varies over time.

We could go to ranges slightly beyond $150 \mathrm{~m}$ and the XOs were still able to ping each other. The range of OLPCs is therefore quite good, though packet losses may actually keep the range to around $60 \mathrm{~m}$ for any practical use. Even $60 \mathrm{~m}$ is quite good to solve the purpose OLPCs were made for - use in a classroom environment.

We summarize our results in Figures 3-5. The error bars in all the three plots represent $95 \%$ confidence intervals. 


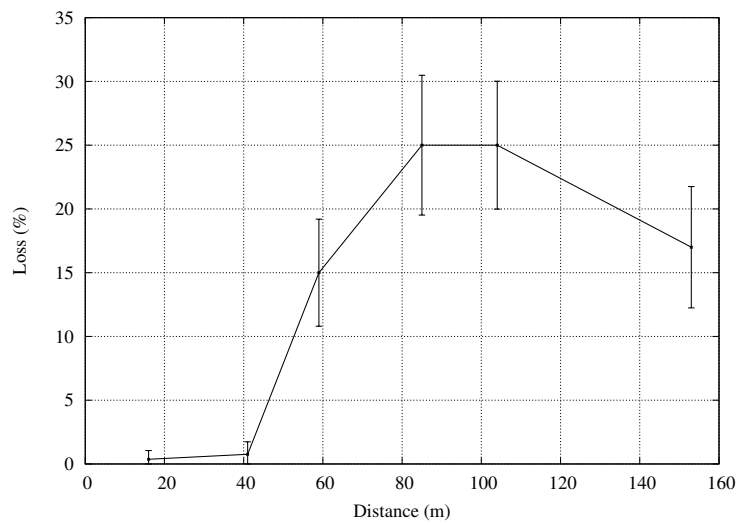

Fig. 4. UDP loss variation with distance

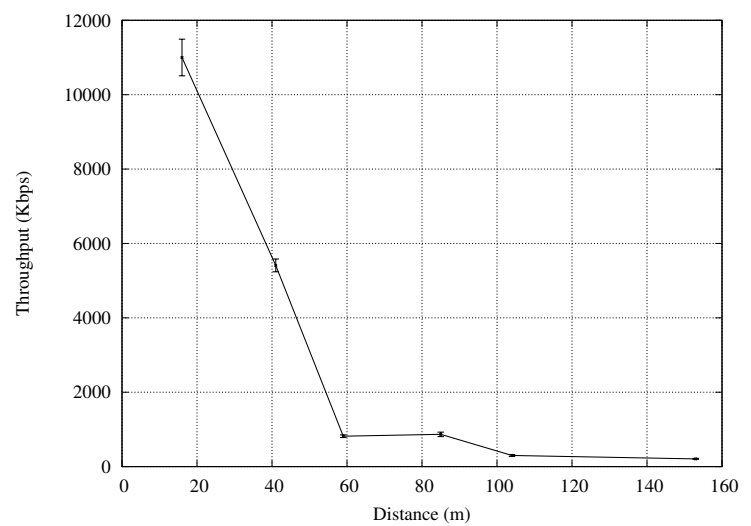

Fig. 5. UDP throughput variation with distance

\section{MULTIHOP EXPERIMENTS}

In a mesh network one can expect data flows to traverse multiple wireless hops from sender to receiver. In this Section we evaluate throughput over multiple hops. All experiments in this section were conducted indoors with distances over a hop varying from one to two meters. Unless otherwise mentioned, all reported throughputs are end to end and per flow.

We received unexpectedly high throughputs in multihop experiments (for example, Figure 8 for topology in Figure 6(b)). One expects the throughput of a flow in a two hop topology (Figure 6(b)) to be about half that in a single hop flow (Figure 6 (a)) since the middle relaying node can either transmit or receive but not do both at one time. This is because the relaying nodes must receive as well as forward data and in theory, each of these takes up half the time available on the channel ( [6] discusses such results in detail). However, our results show this to be not the case. A two hop UDP flow (Figure 8) achieves a throughput average of nearly $10 \mathrm{Mbps}$ (about $70 \%$ of the single hop flow observed in Figure 7).

The nodes select the PHY modulation scheme automatically and give little control over that. To explain the multihop vs direct link behavior one might be tempted to consider that different PHY modulation schemes may have been acting in multihop and single hop scenarios. But our results were the

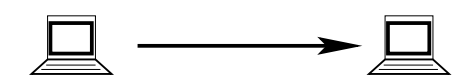

(a) Single hop single flow

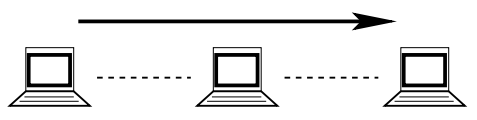

(b) Two hop single flow

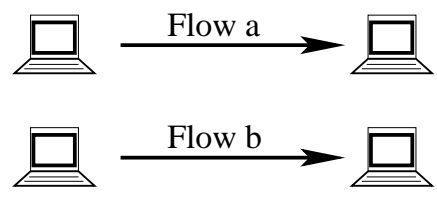

(c) Single hop two flows

Fig. 6. Single and multihop, and single and multiflow topologies

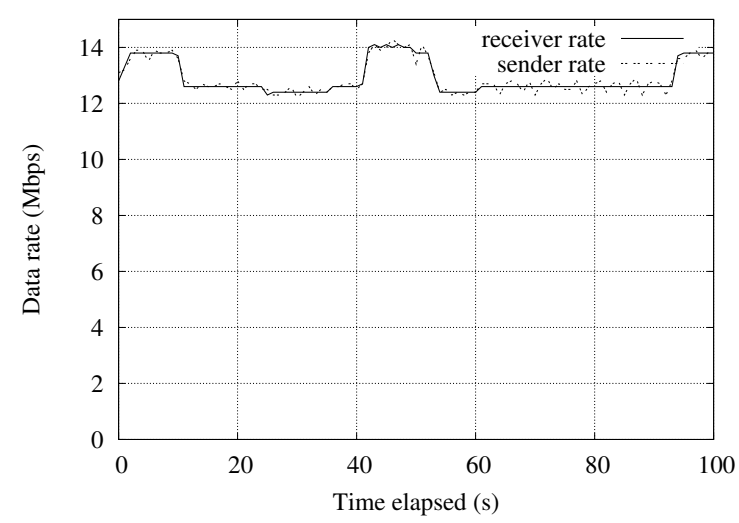

Fig. 7. One hop single UDP flow

same even at very short distances (end to end distance being $1.5 \mathrm{~m}$ ) where one does not expect the modulation scheme to change. Actually a direct hop link consistently showed same throughputs for distances under $1 \mathrm{~m}$ and those around $3 \mathrm{~m}$. Thus varying PHY modulation cannot be the reason for this behavior. In what follows, we perform different experiments to determine the cause of this higher throughput.

We observed that the throughput of a single UDP flow (figure 6(a)) between two XOs does not exceed 12-14 Mbps (Figure 7). However, different pairs of XOs communicating simultaneously while being within transmission range of each other (Figure 6(c)) can achieve a combined net throughput of 22 Mbps (Figure 10). Clearly, the transmission bit-rate on the wireless channel is at least $22 \mathrm{Mbps}$. The results therefore tell us that the upper limit of transmission rate of $14 \mathrm{Mbps}$ is not due to the choice of modulation at the PHY layer using the topologies in Figures 7 and 6(c).

This led us to make a surprising finding that the XOs have different transmission and reception capacities, a phenomenon we term $T x / R x$ asymmetry. We demonstrate it using the two topologies depicted in Figure 9. In the first topology a single $\mathrm{XO}$ receives data from two flows (either UDP or TCP) from two other XOs whereas in the second the direction of data transmission is reversed. All three XOs were within transmission range of each other, with $\mathrm{XO}$-to-XO distances of 


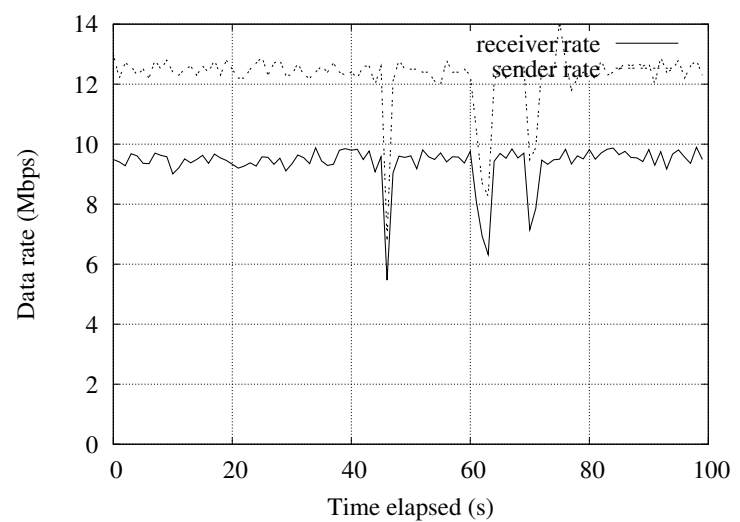

Fig. 8. A single two hop UDP flow

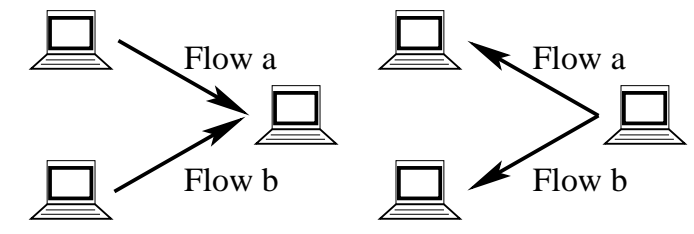

(a) Two senders, one receiver (b) One sender, two receivers

Fig. 9. Setup for testing TX/RX asymmetry

$1 \mathrm{~m}$.

We ensured that the receiver in first experiment is made the sender in second experiment. This avoids errors creeping in due to random uncontrollable changes in the hardware. Also, since the two flows are started asynchronously, we consider data rates averaged over $100 \mathrm{~s}$ rather than the rates in any particular second. Table I gives the throughput obtained from the experiments.

The UDP experiments clearly show that the net rate at which the receiver receives data in Figure 9(a) is more than the net rate at which the sender sends data in Figure 9(b). In the two receivers, one sender case, the total receive rate is limited to at most 13-14 Mbps whereas in the one receiver, two senders case, the total receive rate is around $18 \mathrm{Mbps}$. We conclude from our experiments that:

- Multihop performance is better than expected. The reason for this however is not in PHY modulation scheme selection alone.

- The maximum transmission rate of $14 \mathrm{Mbps}$ cannot be due to the actual choice of modulation level used. Recall that the modulation level sets the maximum physical bit-

TABLE I

AVERAGE RECEIVE RATES (MBPS) FOR THE SETUP AS IN FIGURE 9

(a) Two senders, one re- (b) Two receivers, one ceiver

\begin{tabular}{|l|l|l|}
\hline & UDP & TCP \\
\hline for flow 1 & 7.93 & 4.71 \\
\hline for flow 2 & 9.78 & 3.37 \\
\hline total & 17.71 & 8.08 \\
\hline
\end{tabular}
sender

\begin{tabular}{|l|l|l|}
\hline & UDP & TCP \\
\hline for flow 1 & 6.46 & 4.14 \\
\hline for flow 2 & 6.68 & 4.31 \\
\hline total & 13.14 & 8.45 \\
\hline
\end{tabular}

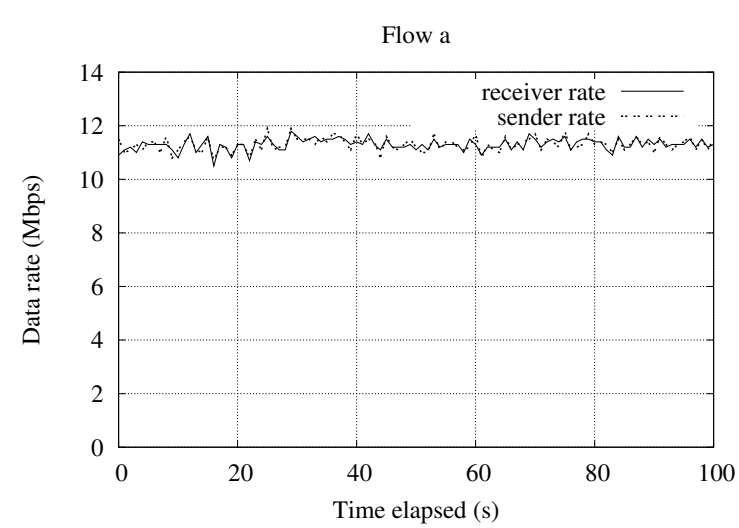

Flow b

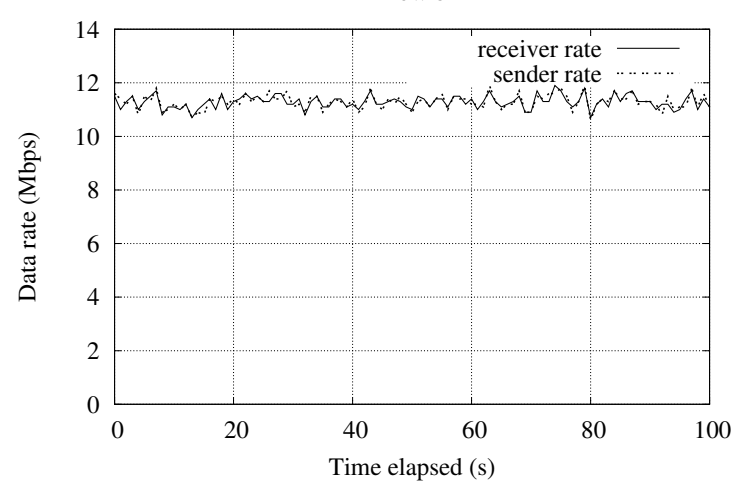

Fig. 10. Two one hop UDP flows

rate of transmission.

- The cause of Tx/Rx asymmetry most likely lies higher than the PHY layer in the protocol stack.

One possible reason for $\mathrm{T} / \mathrm{Rx}$ asymmetry results is that transmission, which involves packet forwarding, is in general more processing intensive than reception. Packet forwarding involves processing like route selection etc. These overheads show up as reduced rates in transmission. Since much of the MAC implementation is inside a closed source firmware, we are unable to do any extensive research as to why a packet reception event needs less processing than a packet transmission event, nor can we modify the routing/forwarding mechanisms. Since the wireless chipsets are responsible for relaying data independent of the main CPU, it is unlikely that the limitations are due to the CPU or the bus. Rather we speculate that it is the chipsets themselves which are responsible for not being able to keep up with sending fast.

Note from Table I that TCP flows do not exhibit Tx/Rx asymmetry unlike the UDP experiment. We explain this by the fact that in the TCP protocol, acknowledgement packets are transmitted in the opposite direction to data packets. Thus all three nodes in the topology of Figure 9 are constrained by processing overheads related to data transmission.

We now explain the unexpectedly high throughputs in multihop experiments using $\mathrm{T} x / \mathrm{Rx}$ asymmetry. A packet reception event consumes much less resources than a packet send event and since the channel has capacity for more transmissions, 


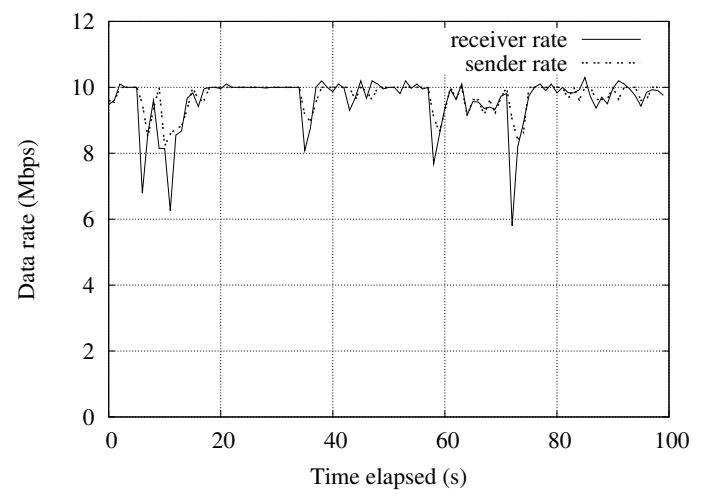

Fig. 11. Single hop UDP flow with RTS/CTS at sending rate $10 \mathrm{Mbps}$

the transmit operation can also be carried out by the intermediate relaying nodes. Because the bottleneck comes while forwarding, the relaying nodes can easily receive and forward data only slightly reducing the throughput in comparison to the single hop throughput.

\section{EFFECT OF RTS/CTS}

The use of RTS/CTS meachanism generally results in a decrease in throughput in both indoor and outdoor settings. Figures 7 and 11 for UDP, and 12 for TCP are results of indoor experiments offering a comparison in throughputs with and without the RTS/CTS mechanism. This is expected in a network where all nodes are close by and there is no hidden node problem which the RTS/CTS handshake seeks to solve. Since RTS/CTS are big overheads in this case, the throughput goes down.

\section{RELATED WORK}

While OLPCs are now influencing the lives of children in developing countries on a huge scale, there exists little literature evaluating the performance of OLPC mesh networking. LOS range of over a kilometer is reported in [7]. Carrano et al. [8] give preliminary results on a performance evaluation of mesh networking in XO's. They have done field tests to evaluate ranges. Their results show considerably lower packet losses (up to 12\%) till a distance of $175 \mathrm{~m}$ beyond which losses rapidly hike to $100 \%$. In our case, OLPCs were not able to ping each other beyond an LOS distance of $153 \mathrm{~m}$. The range may actually vary from machine to machine and perhaps from time to time, depending on the environmental conditions. Hideki et al. [9] have evaluated the impact of RTS/CTS in OLPC mesh networks. Section IV of this paper confirms the findings of their work.

\section{CONCLUSION}

In this paper we have presented our results on OLPC measurements. We find that XOs give high througputs of several Mbps for both TCP and UDP for ranges up to $60 \mathrm{~m}$. In addition, XOs can communicate (albeit with lower throughput) till about $150 \mathrm{~m}$. In other experiments we determined that flows traversing multiple hops obtain higher throughputs than

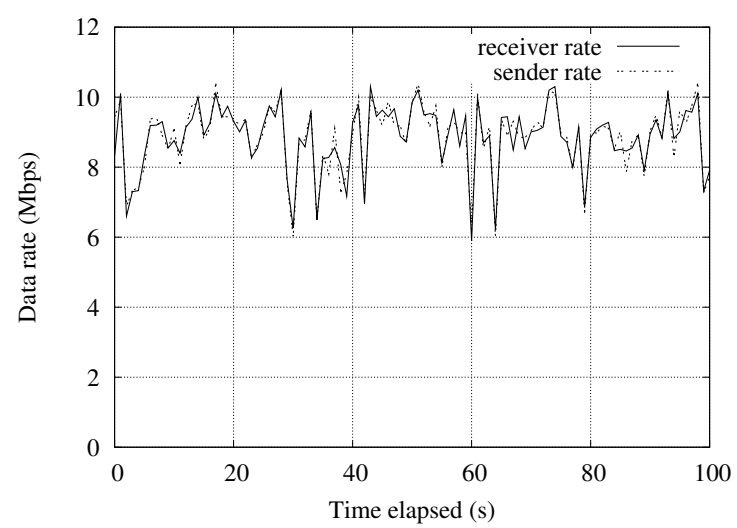

(a) Without RTS/CTS

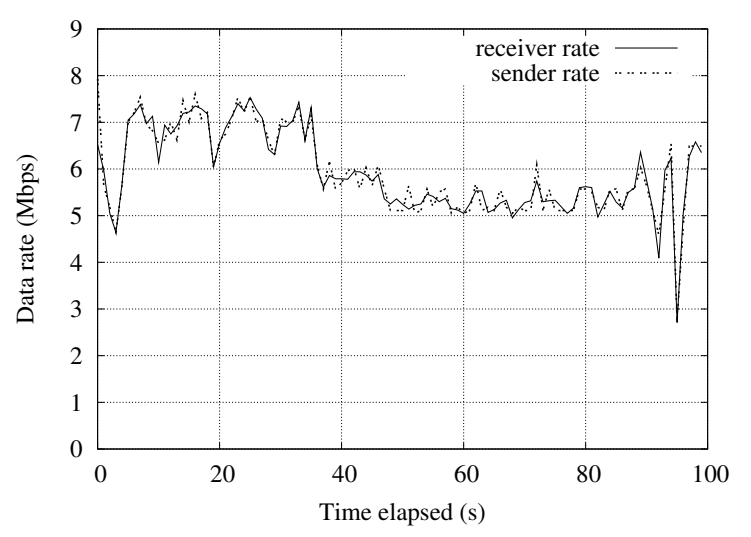

(b) With RTS/CTS

Fig. 12. Single one hop TCP flow with/without RTS/CTS

predicted by theory. We explained this phenomenon through Tx/Rx asymmetry. Finally, we confirmed earlier claims that RTS/CTS overhead reduces throughput in OLPC mesh networks.

\section{ACKNOWLEDGMENT}

This work was supported by Marvell India Pvt. Ltd. (grant number RP02134). We especially thank Sandesh Goel and Ashish Shukla of Marvell for the many enlightening conversations we had with them.

\section{REFERENCES}

[1] I. Akyildiz, X. Wang, and W. Wang, "Wireless mesh networks: a survey," Computer Networks, vol. 47, no. 4, pp. 445-487, 2005.

[2] "One laptop per child (OLPC)." [Online]. Available: http://www.laptop. org

[3] Draft Amendment to standard 802.11: ESS Mesh Networking, IEEE Std. P802.11s/D1.05, June 2007.

[4] C. Perkins, E. Belding-Royer, and S. Das, "Ad hoc on-demand distance vector (AODV) routing," RFC 3561, Jul. 2003. [Online]. Available: http://www.ietf.org/rfc/rfc3561.txt

[5] "Iperf." [Online]. Available: http://dast.nlanr.net/Projects/Iperf/

[6] J. Li, C. Blake, D. De Couto, H. Lee, and R. Morris, "Capacity of ad hoc wireless networks," in Proceedings of the 7th annual international conference on Mobile computing and networking, 2001, pp. 61-69.

[7] "OLPC XO BTest-1 units span a kilometer in WiFi connectivity - One Laptop Per Child News.” [Online]. Available: http://www.olpcnews.com/ hardware/wireless/olpc_xo_btest-1_wifi.html 
[8] R. Carrano, M. Bletsas, and L. Magalhães, "Mesh Networks for Digital Inclusion-Testing OLPC's XO Mesh Implementation," in 80 Forum Internacional de Software Livre, 2007, Porto Alegre. Anais da Trilha Internacional do Workshop do 8 Forum Internacional de Software Livre, 2007.

[9] L. Hideki, R. Martins, A. Guerrante, R. Carrano, and L. Magalhães, "Evaluating the impact of RTS-CTS in OLPC's XOs' Mesh Networks," XXV Simpósio Brasileiro de Telecomunicações (SBrT'07), Recife. Anais do XXV Simpósio Brasileiro de Telecomunicações., 2007. 